\title{
Deformation and fracture properties of pure ice through impact indentation testing
}

\author{
Yuki Nakao $^{1 *}$, Hiroyuki Yamada ${ }^{2}$, and Nagahisa Ogasawara ${ }^{2}$ \\ ${ }^{1}$ Graduate School of Science and Engineering, National Defense Academy, Yokosuka, Japan \\ ${ }^{2}$ Department of Mechanical Engineering, National Defense Academy, Yokosuka, Japan
}

\begin{abstract}
The deformation and fracture properties of ice have attracted considerable research interest. The tip shape of an object that comes into contact with the ice may affect the fracture phenomenon of ice, but these mechanisms have not been elucidated. In previous study, we experimentally showed that the shape of the indenter has a significant effect on pure ice deformation and fracture properties by quasi-static indentation testing. In this study, we focus on the impact fracture of pure ice to clarify the effect of strain rate on deformation and fracture phenomena. The impact indentation test was conducted using direct impact Hopkinson bar method, and a spherical indenter with a diameter of $9 \mathrm{~mm}$ was attached to the tip of the striking bar. The indentation rate was approximately $2.3 \mathrm{~m} / \mathrm{s}$, and the test temperature was approximately $-10^{\circ} \mathrm{C}$. It was clear that the maximum load of the load-displacement relationship was larger than that of the quasi-static indentation testing. This tendency was qualitatively consistent with the compressive strength of the uniaxial compression testing.
\end{abstract}

\section{Introduction}

Ice is one of the well-known materials, and is used in a wide range of applications. On the other hand, ice fracturing causes several problems, such as damage to cars and buildings subjected to hail, collision of sea ice and ships, and falling of ice blocks from aircraft. Ice is also used as a transportation route or runway in certain applications. In this regard, the mechanical properties of ice must be elucidated. The deformation and fracture properties of ice have attracted considerable research interest. The strength of ice has been reported to depend on the temperature, strain rate, and other factors, and several studies have addressed that ice exhibits ductile or brittle behaviour depending on the strain rate [1-3]. The ductilebrittle transition is understood to occur at strain rates from approximately $10^{-4}$ to $10^{-3} \mathrm{~s}^{-1}$.

Here we pay attention to how the ice fractures. Sharp objects such as ice picks are widely used to fracture ice in everyday life. Moreover, ice easily fractures when impacted with the back of metallic spoons. However, the associated fracture mechanisms have not been clarified. Our previous research performed quasi-static indentation testing by conical and spherical indenters, and experimentally showed that the shape of the indenter has a large effect on the deformation and fracture properties of ice [4]. This study focuses on impact

* Corresponding author: em57009@nda.ac.jp 
fracture. We conducted an impact indentation testing using a spherical indenter by the direct impact Hopkinson bar method, and attempted to evaluate the impact deformation and fracture properties of ice.

\section{Material and method}

\subsection{Specimen}

Commercially available pure ice was used as the test material. The pure ice block $(260 \mathrm{~mm}$ $\times 550 \mathrm{~mm} \times 1000 \mathrm{~mm}$ ) was cut into 36 equal parts and processed into a rectangular parallelepiped with a length, width, and height of $20 \mathrm{~mm}, 20 \mathrm{~mm}$, and $30 \mathrm{~mm}$, respectively, using a band saw. The surface of the height direction of the ice was slightly melted on a metal plate and fixed on the specimen mount which was made by an aluminium alloy and acrylonitrile butadiene styrene resin. Then, the specimen was placed on a microtome (REM710; Yamato Kohki Industrial Co., Ltd), and the upper surface of the ice was cut in $10 \mu \mathrm{m}$ steps such that the height became $20 \mathrm{~mm}$. These $20 \mathrm{~mm}$ cubic specimens were used for the experiments.

\subsection{Test method}

The impact indentation test was conducted using direct impact Hopkinson bar method in Fig. 1. The apparatus consists of a striking bar, a supporting bar, a launching device that launches the striking bar by a spring mechanism, coils for measuring velocity and a cooling chamber connected to a cooling system using liquid nitrogen. The tests were recorded using a highspeed camera (MEMRECAM ACS-1; nac Image Technology Inc.) installed in front of the observation window of the chamber, with frame rates of $200000 \mathrm{fps}$. An LED light with a low heating amount was applied from the opposite side of the high-speed camera to pass light through the transparent ice specimen, and the state of crack formation, propagation and fracture was observed as a shadow picture.

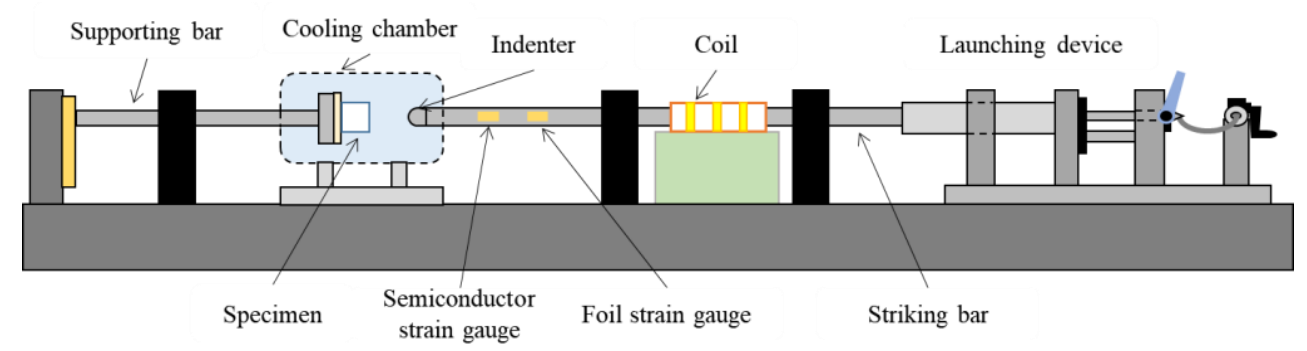

Fig. 1. Schematic of impact indentation test using the direct impact Hopkinson bar method.

\subsubsection{Measurement of load}

The striking bar was a circular tube made of SUS304 with a length of $2000 \mathrm{~mm}$, an outer diameter of $16 \mathrm{~mm}$, and an inner diameter of $12 \mathrm{~mm}$. A spherical indenter with a diameter of $9 \mathrm{~mm}$ was attached to the tip, as shown in Fig.2. Semiconductor strain gauges and foil strain gauges were mounted on the striking bar. The load was obtained by measuring the elastic stress wave generated during indentation using strain gauges and using Eq. (1). 


$$
F(t)=E \varepsilon(t) A
$$

where $E$ is Young's modulus, $\varepsilon(t)$ is the strain wave propagating in the striking bar, and $A$ is the cross-section area of the striking bar.

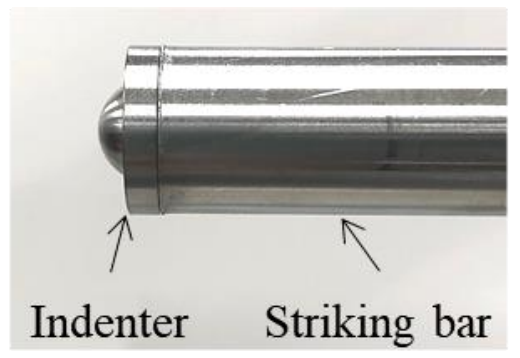

Fig. 2. Photograph of the indenter at the tip of the striking bar.

\subsubsection{Measurement of velocity and displacement}

The impact velocity was measured using a magnet and coils. Three coils with intervals of 10 $\mathrm{mm}$ were placed and passed through the striking bar with the neodymium magnet attached. The velocity of the striking bar was measured using the time difference of the induced electromotive force generated by the magnet passing through the coils. In 12 tests, the average velocity of the striking bar was approximately $2.3 \mathrm{~m} / \mathrm{s}$. The displacement was calculated from the velocity of the striking bar without consideration for the velocity decay due to the low strength of the ice.

\subsubsection{Test temperature}

A chamber (CLC-01; Collet Industrial Co., Ltd.) connecting to a cooling system was used to maintain the low temperature for the test. Nitrogen gas flowed into the liquid nitrogen storage container from the nitrogen cylinder, and low-temperature nitrogen gas was fed to the chamber to cool the inside of the chamber. The flow rate of the nitrogen fed to the chamber was adjusted using a nitrogen gas flow control device (MC-500; Collet Industrial Co., Ltd.). The temperature inside the chamber was measured using a K-type thermocouple and verified using a temperature control device (MC-1000R; Collet Industrial Co., Ltd.). The test was performed at an ice temperature of $-10.1 \pm 0.3^{\circ} \mathrm{C}$, realized after maintaining the temperature in the chamber at $-10 \pm 1^{\circ} \mathrm{C}$ for $15 \mathrm{~min}$.

\section{Results and discussion}

\subsection{Observation of deformation and fracture}

Figure 3 shows the process of ice fracturing obtained by the high-speed camera. The cracks related to fracture grow three-dimensionally inside the specimen. However, it should be noted that this study is a two-dimensional observation because it is taken from one direction. When the indenter came into contact with ice, small cracks were generated in the vicinity of the contact point of the indenter. These cracks hardly propagated until around $30 \mu \mathrm{s}$, but the crack propagation resumed from around $45 \mu \mathrm{s}$, leading to fracture. 


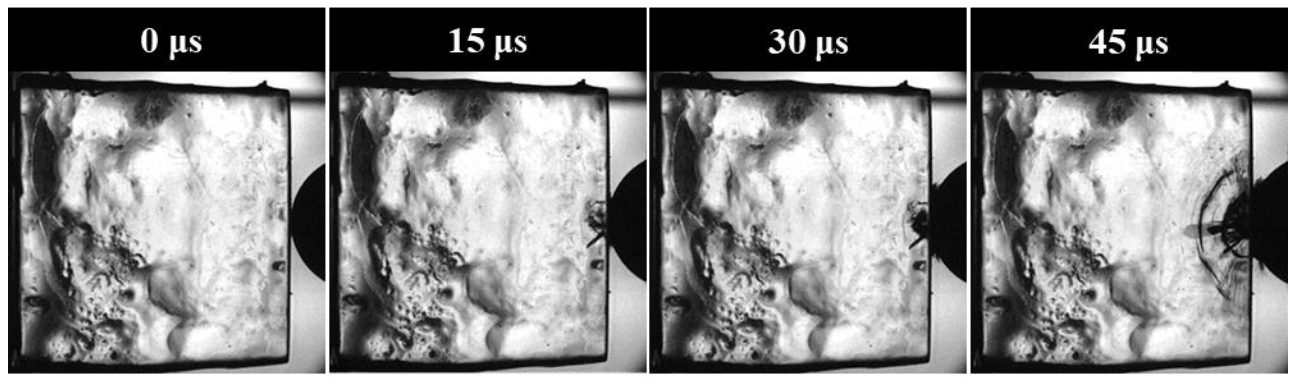

$0.00 \mathrm{~mm}$



$0.144 \mathrm{~mm}$
$0.036 \mathrm{~mm}$

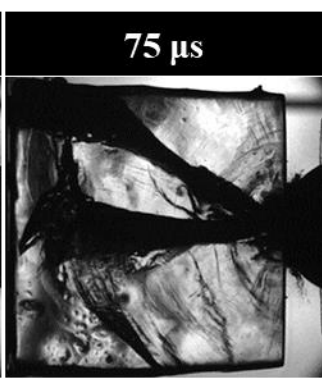

$0.180 \mathrm{~mm}$
$0.072 \mathrm{~mm}$

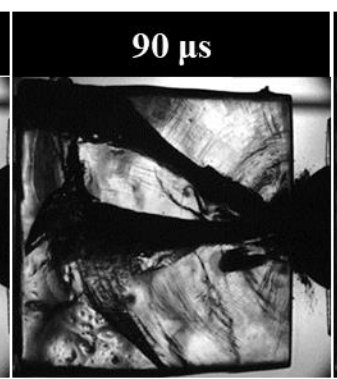

$0.216 \mathrm{~mm}$
$0.108 \mathrm{~mm}$

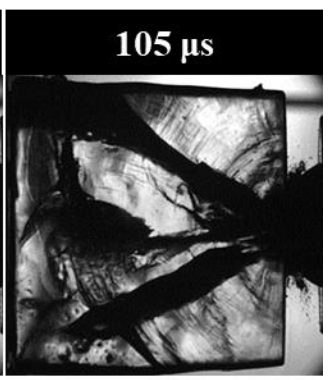

$0.252 \mathrm{~mm}$

Fig. 3. Process of ice fracturing obtained by the high-speed camera. The top of each image shows the time since the ice surface and indenter tip came into contact, and the bottom shows the displacement at that time.

\subsection{Load-displacement relationship}

Figure 4 shows the typical load-displacement relationship in the impact testing. For comparison, the typical result of a quasi-static testing at a displacement rate of $2.0 \mathrm{~mm} / \mathrm{s}$ obtained in previous studies is also shown. When the indenter came into contact with ice, the load increases with the displacement increase, regardless of the indentation rate. In 12 tests, the average maximum load obtained from the load-displacement relationship was approximately $585 \mathrm{~N}$, which was larger than the result of the quasi-static testing (approximately $154 \mathrm{~N}$ ) [4]. It has been reported that the maximum compressive strength of ice in a uniaxial compression test tends to increase in the impact strain rate range in comparison with the quasi-static strain rate [5-6]. The present result suggested that the similar strain rate dependence as in the uniaxial compression testing can be confirmed in the impact indentation testing. Further experiments with different indenter shapes are expected to elucidate the deformation and fracture properties of ice in the impact rate range. 


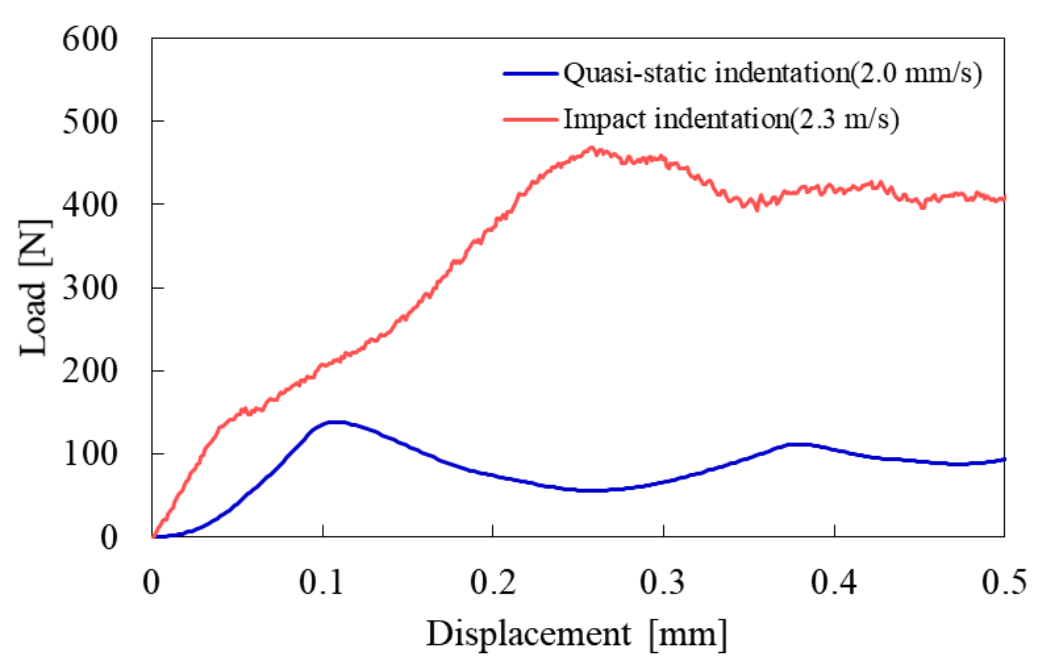

Fig. 4. Relationships between typical load and displacement in impact and quasi-static tests.

\section{Conclusion}

In this study, the deformation and fracture properties of pure ice were evaluated by impact indentation testing using the direct impact Hopkinson bar method. From the high-speed camera image, the state of crack propagation inside the ice specimen and fracture was observed. The maximum load of the load displacement curve was larger than that of the quasi-static testing. Furthermore, the same strain rate dependence of the maximum load of the indentation testing was confirmed as in the uniaxial compression testing by comparing the results of quasi-static and impact testing.

\section{References}

1. I. Hawkes, M. Mellor, J. Glaciol., 11, 103 (1972)

2. L.W. Gold, J. Glaciol., 19, 197 (1977)

3. E.M. Schulson, Eng. Fract. Mech., 68, 1839 (2001)

4. Y. Nakao, H. Yamada, N. Ogasawara, Evaluation of Deformation and Fracture Properties in Ice Using Impact Indentation Testing, in Proceedings of the 13th Symposium for Impact Behavior of Materials, 13-14 October 2020, online (2020)

5. H. Kim, J.N. Keune, J. Mater. Sci., 42, 2802 (2007)

6. M. Shazly, V. Prakash, B. Lerch, Int. J. Soids Struct., 46, 1499 (2009) 